\title{
Searching for the cause of Alzheimer's disease*
}

\author{
Paul J. Harrison, MRC Training Fellow, Department of Anatomy and Cell Biology, \\ St Mary's Hospital Medical School, Norfolk Place, London W2 1PG, and Honorary \\ Senior Registrar, Academic Department of Psychiatry, St Mary's Hospital, \\ London W2 1PG
}

Becoming demented is something people dread most about growing old; the image of a confused and wandering elderly person is a powerful and distressing one. Alzheimer's disease is the commonest cause of dementia, affecting 500,000 people in Britain alone. It is a progressive and presently incurable brain disease, causing great suffering and imposing an enormous burden on health resources. As a result, a large research effort is under way to find out what causes the disease, since a cure is only likely to emerge once the disease is understood. This research is wide-ranging and diverse, as the complexity of Alzheimer's disease requires many lines of enquiry if the puzzle is to be solved. At one extreme, the research focus is upon large groups of elderly people who are being followed up to see what features predict and herald the onset of dementia. At the other, individual genes, molecules and brain cells are under detailed investigation. Some of the latter studies have given important clues as to the cause of Alzheimer's disease, and these are briefly mentioned here.

Sometimes Alzheimer's disease runs in families, suggesting that an abnormal gene is being passed on from one generation to the next. Genes are the instructions contained in every cell, used to make the protein molecules which determine how we develop and which control the activities of organs like the brain. A defective gene can therefore stop the brain working properly, and contribute to the loss of memory and personality changes which afflict demented people. We do not yet know what this 'Alzheimer's gene' might be, nor how it may exert its effects, but rapid progress is being made towards identifying the culprit. Once it has been found, it may be possible to identify members of families with the gene who are at high risk of developing the disease, as well as to modify the gene and prevent the disease occurring.

In most cases, however, Alzheimer's disease seems to strike at random and it is unlikely that a defective gene is responsible. Another avenue of research is to study how brain cells use their genes: even if the genes

* The author of this paper was chosen as the inaugural winner of the Morris Markowe Public Education Prize in March 1990. themselves are normal, perhaps Alzheimer's disease arises because cells wrongly process the genetic information. To illustrate, having a defective gene is equivalent to a faulty typewriter key: the wrong letter will be produced even if the typewriter is used correctly. Wrongly processing a gene is like having a perfect typewriter but a typist who makes various errors. The outcome in both cases will be to produce incorrect copies of the instructions; the question is whether Alzheimer's disease arises from the typewriter or the typist.

Mistakes in the use of genes are important because they lead to abnormal proteins being produced. These can interfere with the working of brain cells and may result in the mental problems seen in the disease. For example, brain cells have systems for importing and exporting protein molecules and for moving them around within the cell. If a defective protein is produced it may block these systems, preventing the cell working properly and causing it to die. Indeed, there is evidence that mistakes of this kind do occur in the processing of some genes and may play an important role in the disease. Similarly, some proteins may be made in the wrong place, while others are produced in the wrong quantity. One diffculty in this research is that so many brain cell activities and proteins become affected by Alzheimer's disease that it is hard to separate the crucial initial events from the many secondary effects which follow on. It seems that by the time symptoms of dementia appear, the brain is already very sick, and the earliest changes have occurred several years before.

A final question concerns what first sets off these problems and actually causes the disease to start. A number of candidates are being investigated, including toxic substances such as aluminium or a virus. One theory suggests that the agent enters the brain by way of the nose or maybe the bloodstream; it then accumulates inside the brain cells over many years and results in the abnormal proteins being produced, eventually damaging the brain to the point where dementia begins.

The wide range of research into Alzheimer's disease promises much in the next decade, but also indicates that so far it is not clear as to where the breakthrough will occur. It may be the genes themselves or their 
processing, the build-up of a toxin, or most likely a combination of several factors, that finally produces the disease. Until more is known about these processes it is unlikely that effective treatments will become available, and the sporadic sensational reports of 'a cure for Alzheimer's disease' are premature. Finally, the research into Alzheimer's disease, which is paralleled by similar approaches to other mental illnesses, also demonstrates how far psychiatry has moved towards being part of modern scientific medicine, viewing such diseases as physical disorders of the brain rather than as social or psychological problems. However valuable this may be, we must not allow the emphasis on molecules to replace our concern for people, for psychiatry will always need to attend to both equally.

\title{
The assessment of parenting: some interactional considerations
}

\author{
Peter Reder, Consultant Child Psychiatrist, Charing Cross Hospital, 2 Wolverton \\ Gardens, London W6; and CLARE LUCEY, Lecturer in Forensic Child \& Adolescent \\ Psychiatry, Westminster Children's Hospital, Vincent Square, London SW1
}

Psychiatrists are frequently invited to give expert opinions to Courts about children's emotional welfare, their parents' capacity to care for them and issues of placement and access. Previous child abuse/ neglect may have been suspected or confirmed or the parents might suffer from psychiatric symptoms. Assessment may be required of parents' mental state with a guide to prognosis, but, in addition, factors in parent-child relationships will be crucial to overall assessments of parenting.

From our perspective as child psychiatrists we shall present the framework that guides our Court assessments. This framework is not intended as a checklist or rating scale of 'normal' behaviour, but it suggests a range of themes to be assessed, some of which may be more relevant than others in any particular case. It is equally applicable to fathers, mothers or other caretakers.

\section{An interactional framework}

The factors we assess can be grouped into four, interrelated clusters: the parent's relationship with the child; the parent's relationship to the role of parenting; influences from family relationships; and relationships with the external world.

\section{The parent's relationship to the child}

The most obvious area to explore is the parent's feelings towards the child and the basis for them. In addition, the degree to which the parent enacts those feelings is important, especially if they are of hostility or rejection.
Another facet is the degree of psychological merging or differentiation between the parent and child, since the child's development requires appropriate parental responses along this continuum. Thus, some merging is involved in empathy and allows the parent to show concern for, and interest in, the child's wellbeing and experiences. It also allows the parent to put him/herself in the child's place, appreciate the child's perspective on experiences and events and acknowledge how the child must have felt when subjected to abuse or family disharmony in the past.

On the other hand, persistent lack of differentiation will draw the child into parental psychopathology and inhibit the child's psychological development and acquisition of autonomy. Therefore, we assess whether the child is viewed as a person in his/her own right, with feelings, ideas, and an identity that is different from the parent. The child's needs must be recognised, differentiated from the parent's and given primacy over them. One way that the parent can demonstrate this is by anticipating the child's needs for protection in the future and endeavouring to minimise the child's exposure to potentially dangerous situations. For example, a mother may have to chose between the security of her abused child and loyalty to the abusing partner.

\section{The parent's relationship to the role of parenting}

A number of authors have described the basic needs of children that their parents must satisfy (e.g. Kelmer Pringle, 1978; Adcock \& White, 1985). These needs differ according to the child's age and include adequate antenatal care in anticipation of the baby, 